\title{
"SHOPPING" À LA FRANCAISE: APPROVISIONNEMENT ALIMENTAIRE ET SOCIABILITÉ
}

\author{
Sophie Chevalier \\ Université de Franche-Comté - France
}

Resumé: L'auteur décrit les pratiques d'approvisionnement alimentaire des habitants de trois villes françaises, Paris, Lyon et Besançon, en les contrastant avec des banlieusards. Elle montre que ces citadins des centres villes fréquentent les commerces alimentaires de proximité L'analyse de ces pratiques révèlent que c'est la dimension sociale qui est la plus importante à examiner pour comprendre ces pratiques d'approvisionnement, en lien avec la sociabilité de quartier. C'est la possibilité de créer des liens personnalisés entre commerçant et client, et "d'être un client reconnu comme habitant d'un quartier". Les commerces "font" la vie de quartier pour la plupart des interlocuteurs des centres villes étudiés. Il existe donc à la fois une sociabilité et une socialisation autour de l'activité d'approvisionnement: "faire ses courses" crée du lien social.

Mots-clefs: approvisionnement alimentaire, centre ville, petit commerce, sociabilité.

Resumo: A autora descreve as práticas de abastecimento alimentar doméstico de habitantes de três cidades francesas, Paris, Lyon e Besançon, contrastando-as com aquelas dos moradores dos subúrbios. Ela mostra que aqueles que vivem nas áreas centrais das cidades freqüentam os pequenos estabelecimentos comerciais de gêneros alimentícios da proximidade. A análise dessas práticas revela que a dimensão social é a mais importante a ser examinada para compreender essas práticas de abastecimento, relacionadas com a sociabilidade de vizinhança. Trata-se da possibilidade de criar laços personalizados entre comerciante e cliente, e de "ser um cliente reconhecido como morador do bairro". Os estabelecimentos comerciais que "fazem" a vida do bairro para a maioria dos interlocutores das áreas centrais da cidade estudados. Existe então, ao mesmo tempo, uma sociabilidade e uma socialização em torno da atividade de abastecimento alimentar doméstico: "fazer as compras" cria o laço social.

Palavras-chave: abastecimento alimentar doméstico, centro, pequeno comércio, sociabilidade. 
Un visiteur étranger qui se promène dans une ville française, un dimanche matin, ne manquera pas d'être intrigué par la queue des clients devant une boulangerie-pâtisserie ou au marché, chez le traiteur, le boucher ou le fromager. Ces scènes, attribuées plutôt à une économie planifiée de pénurie, sont-elles significatives des pratiques d'approvisionnement des citadins de ce pays? Va-ton réellement “faire ses courses” dans des commerces spécialisés de quartier quotidiennement? Ou n'est-ce qu'une distraction du week-end?

Certes, vue de l'extérieur, par exemple de Grande-Bretagne, la France est le pays du petits commerces dont la pérennité est assurée par une législation favorable (Péron, 1998). Par contraste avec l’Angleterre qui, si elle est aussi le pays des petits commerçants, du moins, dans un certain discours politique, et bien que le premier magasin moderne soit britannique (Brewer; Plumb, 1982; Mc Kendrick), ne l'est plus depuis longtemps. Les premières grandes surfaces - supermarchés et hypermarchés ${ }^{1}$ - s'implantent en France dans les années 1960, et gagnent peu à peu des parts du marché: en 1994, 64\% des Français y achètent leurs produits alimentaires. Ceux qui ne fréquentent pas ces commerces se rencontrent surtout chez les Parisiens qui font leurs achats dans les petits commerces et sur les marchés (Régnier; Lhuissier; Gojard, 2006, p. 27ss).

L'observation des commerces en tant que tels et leur intégration dans le tissu urbain a été étudié dans de nombreux travaux anthropologiques (Bouly de Lesdain, 1999; Brody, 1987; Lallement, 2005; Raulin, 2000). Les analyses de Michèle de La Pradelle (1996, 2002) ont montré que les relations marchandes engendrent des champs de rapports sociaux spécifiques et que les pratiques d'approvisionnement participent de la sociabilité d'un quartier.

Ici, nous nous intéresserons tout d'abord, à décrire les pratiques d'approvisionnement des ménages qui habitent des quartiers des centres villes de Paris, de Lyon et de Besançon, et aussi un certain nombre de familles qui vivent dans les banlieues parisiennes et lyonnaises. ${ }^{2}$ Ces dernières nous

\footnotetext{
${ }^{1}$ Un supermarché a une surface comprise entre 400 et $2.500 \mathrm{~m} 2$ et un hypermarché a plus de $2.500 \mathrm{~m} 2$.

2 Cette recherche fait partie d'une étude plus vaste intitulée "Etude internationale sur les modes d'habitat" qui a été commanditée par le Plan, Urbanisme, Construction et Architecture (PUCA) à l'association "Pollen". Le terrain d'enquête a été mené en 1999-2000 (Chevalier, 2000). De plus certains des matériaux et des analyses présentés ici ont fait l'objet d'un article "'Faire ses courses en voisin': pratiques d'approvisionnement et sociabilité dans l'espace du quartier”, www.revuemetropoles.com, n. 1, à paraître en 2007. Nous avons rencontré 35 familles: tous les ménages sont composés d'un couple (conjoints mariés ou concubins) avec au moins un enfant. Ils vivent soit dans
}

Horizontes Antropológicos, Porto Alegre, ano 13, n. 28, p. 65-86, jul./dez. 2007 
permettront de contraster nos données sur les pratiques des résidents des centres villes. Cette description permettra, dans un deuxième temps, de comprendre l'insertion des acteurs dans leur environnement de proximité. "Faire ses courses" dans son voisinage est pris comme un révélateur d'une intégration éventuelle des ménages dans un quartier, puisque s’approvisionner permet de construire un rapport à son environnement, un lien qui soit à la fois physique (parcours de l'espace), social (liens sociaux de voisinage) et symbolique (représentations de son quartier). ${ }^{3}$

Un anthropologue britannique a mené une étude similaire portant sur une rue londonienne (Miller, 2005): il y montre la façon dont les classes moyennes construisent une représentation imaginaire des petits commerces de leur quartier. Même si elle n'est pas la seule recherche existante dans ce domaine, elle nous servira de contrepoint pour notre analyse du cas français, tout d'abord parce qu'elle porte sur une classe sociale similaire habitant un milieu urbain et que l'auteur utilise une approche méthodologique semblable à la nôtre. Mais aussi, parce que les analyses du rapport à l'alimentation et à la cuisine se sont inscrites très souvent dans cette opposition heuristique entre les deux sociétés, françaises et britanniques (Goody, 1982, Mennell, 1986).

L'insertion locale d'un ménage possède une double dimension: on pourrait la qualifiée tout d'abord, d'objective, par la pratique d'un espace, la fréquentation des commerces, des installations de quartier et de ses voisins; mais aussi subjective, c'est-à-dire la façon dont les acteurs se représentent et vivent cette intégration dans le quartier. Bien évidemment elles sont intimement liées.

les centres villes et soit dans les banlieues, sauf à Besançon où la taille de la ville ne justifie pas un tel choix. Ainsi, à Paris, quatre ménages vivent dans le IIIème arrondissement, quatre dans le Xxème arrondisssement et quatre en banlieue parisienne; à Lyon, cinq ménages habitent à La Croix-Rousse (Ivème arrondissement), deux à Saint-Georges (Vème), un sur la presqu'île et trois à Oullins. A Besançon, quatre ménages résident dans le centre historique ("La Boucle”), et huit autres en dehors, mais toujours en centre ville. D’un point de vu méthodologique, nous avons pu conduire plus d'entretiens que d'observations directes. Les entretiens ont été conduits auprès du couple, à leur domicile. Les informateurs ont été contactés par un système de "boule-de-neige".

${ }^{3}$ Il est vrai cependant que l'on assiste actuellement à la transformation rapide de ces usages avec l'irruption des achats alimentaires par internet: c'est-à-dire la "délocalisation" des courses ou plutôt “l'a-localisation”, l'absence d'ancrage dans l'espace. Nous n'en développerons pas ici l'analyse, mais il était important de le mentionner comme facteur de changements possibles des habitudes d'approvisionnement, et par là même, du rôle des commerces dans la sociabilité de quartier.

Horizontes Antropológicos, Porto Alegre, ano 13, n. 28, p. 65-86, jul./dez. 2007 
Néanmoins, pour que la première se réalise, il faut que les acteurs puissent se représenter leur vie dans un espace, si possible de manière positive. ${ }^{4}$

\section{Choisir son voisinage}

Nos informateurs font partie de groupes sociaux ${ }^{5}$ qui ne sont pas soumis à de très fortes contraintes financières dans le choix de leur logement; ainsi leur stratégie résidentielle est liée à celui d'un “mode de vie” et à une sorte de “représentation sociale” idéale du quartier (Desjeux; Monjaret; Taponier, 1998).

La possibilité de se déplacer à pied pour ses courses quotidiennes ou de se distraire grâce à la proximité de cafés et de magasins fait de leur environnement direct des lieux appréciés pour leur animation par tous les ménages citadins rencontrés. Les habitants du IIIème arrondissement parisien, du IVème et du Vème arrondissement lyonnais ou du centre ville de Besançon citent aussi volontiers la dimension esthétique et architecturale de leur quartier. L'expression de ce choix résidentiel est particulièrement vraie pour les quartiers qui ont une “image forte”, bien spécifique dans une ville, comme Le Marais à Paris, La Croix-Rousse ou Saint-Georges à Lyon ou encore certaines rues à Besançon. Dans cette ville, la taille du centre permet plus de souplesse dans la façon dont on construit son appartenance, qui va d'une seule rue à l'ensemble de la "boucle". ${ }^{6}$ Dans les villes plus grandes, nos interlocuteurs explicitent leur localisation en distinguant très clairement leur appartenance à un quartier plutôt qu'à un autre.

Cette élection d'un “mode de vie” correspond avec la représentation sociale du quartier: des résidents-voisins qui auraient les mêmes intérêts que nos informateurs et qui, d'une certaine manière, seraient leurs semblables socialement et culturellement. Cette idée du “village dans la ville”, pour paraphraser le titre de l'ouvrage pionnier de Young et Willmott (1957/1983), contient aussi celle de société d'inter-connaissance, impliquant une certaine homogénéité sociale. C’est la possibilité de rencontrer ses semblables, qui séduit nos interlocuteurs, plus

\footnotetext{
${ }^{4}$ Il faut remarquer que cette représentation positive est d'abord pour soi; cependant, l'image que les autres peuvent vous renvoyer de votre quartier, si elle est négative, et même si vous vous y trouvez bien, conduit à reconsidérer sa propre représentation.

${ }^{5}$ Seuls deux ménages vivent dans des logements HLM (Habitation à Loyer Modéré) dont les loyers sont donc contrôlés et plus bas que ceux du marché. Par ailleurs, dix-huit familles sont propriétaires de leurs logements sur les trente-cinq ménages enquêtés.

${ }^{6}$ Partie de la vieille ville de Besançon enserrée dans une boucle du Doubs, désignée communément par le terme "la boucle".
} 
que la pratique réelle d'une sociabilité de quartier. Cette similitude sociale désirée peut inclure bien sûr un certain mélange ethnique et sociale qui donnerait cet aspect varié et distrayant à certains quartiers. Mme Sillard ${ }^{7}$ décrit son environnement dans le XXème arrondissement parisien:

C'est un quartier que j'aime bien parce que c'est un mélange populaire et bourgeois. C'est vrai qu'il y a des mélanges ethniques aussi mais pas avec les problèmes majeurs qu'on peut rencontrer dans des parties plus défavorisées du quartier [...] Ce quartier m'a permis finalement de ne pas être désorientée par rapport à ce qui fait partie de mon éducation, parce que je continue quand même à côtoyer des gens, pas socialement mais culturellement proches de moi, intellectuellement proches de moi, par contre je fréquente des milieux sociaux très différents.

“Habiter avec ses semblables” permet d'espérer se lier d'amitié avec ses voisins, et dans les faits, conduit nos informateurs à avoir souvent leurs amis à proximité, venus se regrouper peu à peu dans le quartier. Cette présence amicale ou de membres de la famille peut d'ailleurs constituer de bons motifs dans le choix du quartier, surtout pour des ménages qui arrivent dans des villes qu'ils ne connaissent pas. Construire des relations de voisinage avec ses "semblables", ou la présence d'amis ou de parents, tout ceci concourt à valoriser les lieux où l'on vit et à guider les choix résidentiels. Une fois installés dans tel ou tel endroit, comment nos informateurs définissent-ils les limites géographiques de l'espace qu'ils considèrent comme leur “quartier”?

Dans son enquête à Londres, Daniel Miller (2005) montre une analogie entre le "quartier" et la rue: nous la retrouvons dans l'affirmation d'un de nos informateurs: "Mon quartier, c’est ma rue”. La rue est donnée pour le quartier, car c'est à travers elle que les Britanniques penseraient la ville (Miller, 2005, p. 19). Il existe selon l'auteur, une véritable “idéologie de la rue” en GrandeBretagne qui ne trouverait pas d'équivalent ailleurs, sauf peut-être aux EtatsUnis (Hannerz, 1969; Whyte, 1934-1993). ${ }^{8}$ Elle suscite imaginaire et

\footnotetext{
7 Tous les noms de nos informateurs ont été changés.

${ }^{8}$ Ainsi les trois séries les plus populaires de la télévision britannique qui sont programmées depuis plusieurs décennies, plusieurs jours par semaine, qui ont pour décor des quartiers ouvriers et dont les lieux de la fiction, sont, par excellence, la rue: Coronation Street à Manchester, East Enders à Londres et Brookside à Liverpool. Si ces séries n'ont pas traversé la Manche, en revanche, de manière plus ludique, la série pour enfants, "Rue Sésame”, d'origine américaine, est présentée sur les chaînes françaises. Il serait très intéressant de comparer ces représentations de la "rue” britannique et française, avec la “rua” brésilienne telle qu’elle s’oppose à la “casa” (DaMatta, 1984, 1985).
}

Horizontes Antropológicos, Porto Alegre, ano 13, n. 28, p. 65-86, jul./dez. 2007 
représentations qui sont autant de ressources pour penser la ville, pour penser le quartier, sans nécessairement s'articuler avec les pratiques de ses habitants.

A Paris, à Lyon, ou dans une petite ville comme Besançon, nos informateurs ont une idée des délimitations "physiques" (Seine, boucle du Doubs, Saône ou Rhône) et administratives du quartier, mais "leur" quartier constitue toujours une entité spatiale plus réduite, le plus souvent celle d'une ou de quelques rues. Les limites géographiques fixent souvent les “frontières" que l’on ne dépassera pas à pied. Ces déplacements piétonniers créent des cercles dont le point central est le logement, avec des limites extrêmes qui sont souvent des "frontières physiques" (ponts; fleuves; etc.). Ce parcours physique de l'espace permet, non seulement de décrire son “quartier” et ses limites, mais aussi son appropriation. Il constitue la grande différence avec les habitants des zones de pavillons de la banlieue parisienne que nous avons interrogés. En effet, ceux-ci utilisent leurs voitures pour leur déplacement. "Leur” quartier comprend au plus les quelques maisons du voisinage et parfois l'allée dans laquelle est situé leur propre pavillon. La banlieue apparaît alors comme un espace plus complexe que celui des centres villes, nécessitant une connaissance qui ne passe pas par une pratique physique directe de celui-ci (piétonnier).

Outre cette dimension, la délimitation de l'espace est liée à celle de proximité sociale. Les habitants de la Croix Rousse à Lyon distinguent nettement le "Plateau” des “Pentes”: “Quand les gens disent qu'ils viennent de la Croix Rousse, on leur demande si c'est des Pentes ou du Plateau. Les Pentes, c'est considéré comme le quartier pauvre par rapport au Plateau, alors que le Plateau est devenu 'top'”, confirme M. Baudard (qui vit sur le Plateau). Ce dernier commentaire rejoint ce que nous avions déjà observé: plusieurs critères sont utilisés pour définir ses frontières dont l'un est celui de la proximité sociale. Mais laissons la parole à Mme Sillard, habitante de la rue St Fargeau, dans le XXème à Paris: “[...] la limite, elle est très simple, c’est la rue St Fargeau. Donc en fait on est protégé” - dans son idée d’une population trop mélangée ethniquement et socialement - qui rend l'environnement “craignos” selon ses termes, et continue-t-elle "et en même temps on côtoie toute une population qui est une population de notre époque”. Sa perception est partagée par d'autres résidents de cet arrondissement qui en fixent les limites à la rue près, en lien avec la composition sociale de la population. 
Parcouru à pied, le quartier est un espace d'interconnaissances et de voisinage: pouvoir être salué comme un habitant du lieu, être reconnu par les autres résidents et par les commerçants constituent autant de preuves tangibles de son appartenance à un quartier qui se réduit souvent à une rue. Même si les habitants de la rue Rambuteau à Paris fréquentent d'autres coins de leur arrondissement, cette rue est "leur quartier”. Bruno Magnanat considère que le sien est la rue Bersot à Besançon, bien qu'il se déplace à pied dans toute la “boucle”. Il y connaît tous les commerçants, les propriétaires des nombreux cafés, qui le saluent au passage.

L’inscription des ménages dans l'espace urbain commence par leur choix résidentiel, fait en recherchant une adéquation entre un mode de vie et une aspiration sociale. Ce lien constitue une condition à l'élaboration d'une sociabilité de quartier. D'une manière générale, on constate que l'intégration d'un ménage dans un quartier est lié à des processus d'appropriation sociaux, symboliques et physiques de celui-ci qui débutent avec le choix du lieu de vie et qui sont réactivés dans des pratiques de le vie quotidienne. Parmi ces pratiques, celles liées à l'approvisionnement semblent être devenues, ainsi que les commerces eux-mêmes, des “ressources symboliques pour penser la rue” (Miller, 2005, p. 19), en d'autres termes le quartier.

\section{Aller "faire ses courses"}

Dans sa recherche londonienne, Miller (2005) a montré que la plupart de ses informateurs issus des classes moyennes ne fréquentait pas ou en “dépannage” les magasins de proximité, malgré un discours de soutien affiché en faveur des petits commerces. Ils préfèrent prendre leur voiture pour aller dans des galeries commerçantes dans la périphérie plus éloignée. Seuls les résidents les plus modestes étaient contraints de s’approvisionner dans la rue, en regrettant l'absence de supermarché et de centre commercial. L'auteur conclut que "seuls deux commerces sur vingt ont véritablement réussi à créer un 'sens de la rue': le coiffeur et le quincailler” (Miller, 2005, p. 20). Il convient de remarquer que ces deux commerces ne sont pas des magasins d'alimentation.

Dans notre recherche, nous ne sommes pas partie à la quête du/des commerces qui donneraient sens à l'environnement de proximité de nos interlocuteurs. Dans un premier temps, nous nous sommes surtout attachée à décrire les pratiques d'approvisionnement, "faire ses courses”, à reconstituer 
la provenance des marchandises, les rythmes des achats, et qui dans le ménage les prend en charge. ${ }^{9}$

Parmi les aliments, deux semblent posséder un statut particulier qui requiert de s’approvisionner dans des commerces spécialisés, autres que dans des super ou hypermarchés: le pain et la viande. Ces deux aliments sont des éléments importants de la diète alimentaire (Kaplan, 1996, 2006; Mintz, 1996, p. 96ss); certes la consommation de pain a diminué depuis trente ans, mais la quantité consommée reste, statistiquement, de 54 kg par personne en 2003 (Régnier; Lhuissier; Gojard, 2006, p. 25). Les boulangeries conservent un peu plus des trois-quart du marché du pain (Régnier; Lhuissier; Gojard, 2006, p. 29). Quant à la viande, elle occupe encore le premier poste des dépenses alimentaires environs un quart - du panier de la ménagère. ${ }^{10}$

Ces boulangeries et boucheries sont souvent situées dans le quartier, voire dans leur rue, et les clients peuvent s'y rendre à pied. Cependant, le pain est aussi un achat qui se fait souvent soit autour de son lieu de travail, soit sur le chemin du retour, en fin de journée. Les autres achats alimentaires, comme l'épicerie, se font dans des supermarchés de proximité (sauf pour les ménages de banlieues qui n’ont pas toujours cette possibilité) pour des raisons de transports, mais aussi de qualité des produits. Patrick Gouriard et Agnès Onnard ne consomment que des produits biologiques qu'ils trouvent dans un supermarché spécialisé de leur quartier, le Marais. On note aussi la persistance de la fréquentation des marchés ouverts de fruits de légumes au moins une fois par

9 Sur les trente-cinq ménages interrogés, douze possèdent une résidence secondaire. Parmi les vingttrois familles qui n'en ont pas, la moitié a accès d'une manière ou d'une autre à un autre logement appartenant à sa parenté, en général à la campagne. On observe le maintien d'une certaine autoproduction alimentaire: en effet quelques ménages cultivent, du moins en partie, leurs fruits et légumes dans leurs jardins. Parfois ceux-ci sont attenants à leur logement lorsqu'ils vivent endehors des centres villes, mais souvent ce sont des informateurs qui ont une résidence secondaire (Chevalier, 1999). Quelques jeunes couples, surtout bisontins, reçoivent des fruits et des légumes, mais aussi de la viande (volailles et lapins, par exemple) de leur parenté rurale. Ces produits constituent une partie importante de leur approvisionnement alimentaire. D’autres ménages, qui ont de la famille en province, reviennent de leurs séjours la voiture pleine de bocaux et conserves. Cette circulation est une forme d'aide de la part des parents qui s'inscrit dans les transferts générationnels.

${ }^{10}$ On peut poser l'hypothèse que le choix d'un commerce spécialisé pour l'achat de la viande est liée aux problèmes liés à l’ESB (encéphalopathie spongiforme bovine) dite "maladie de la vache folle".

Horizontes Antropológicos, Porto Alegre, ano 13, n. 28, p. 65-86, jul./dez. 2007 
semaine (en général le week-end). ${ }^{11}$ Plus de la moitié des ménages interrogés dise faire leurs courses de fruits-légumes sur les marchés hebdomadaires. Cette rapide description des pratiques montre que la diversité de l'offre commerçante est essentielle dans un quartier: supermarché, commerces spécialisés et marchés. Elle permet de satisfaire la plupart de ses besoins à proximité de son domicile. Sortir de son quartier urbain pour ses courses est lié à des raisons diverses, qui sont économiques comme dans l'exemple précédent, ou encore la recherche de produits spécifiques, liés à des pratiques religieuses ou dont la qualité est réputée supérieure. La famille Kehour, d'origine algérienne, se déplace dans le XIème arrondissement pour aller se servir dans une boucherie halal: "Pour la viande, on a un boucher que l'on connaît par la famille qui achète là-bas. Ma tante achète toujours chez lui. C'est un peu la boucherie de famille!”. Jocelyne Sillard va parfois rue des Rosiers pour de la pâtisserie, surtout au moment des fêtes juives. Cependant, plus que les produits alimentaires particuliers, ce sont souvent des liens personnels noués avec le commerçant qui vont conduire certains informateurs à se déplacer pour le suivre dans ses déménagements, comme l'explique Robert Choron:

On se déplace pour la boucherie. Parce qu'il se trouve qu'on allait dans la boucherie au centre ville. Le jeune boucher qui nous servait, on le trouvait éminemment sympathique; parce qu'on aime bien, Christine comme moi, parler avec lui. On aime bien avoir le contact. Ce boucher est parti de cette boucherie. Il est parti dans un premier temps dans le Haut-Doubs. Un jour en se baladant, on a même été acheter de la viande. Il s'est installé maintenant dans le quartier de Saint-Ferjeux. On y va en voiture pour faire des courses pour la semaine.

M. Baudard a deux bouchers: "Il y en a un que je connais qui est un ami et qui est de très bonne qualité, mais qui n'est pas sur mon trajet. Il y en a un autre qui est sur le trajet de l'école. Donc je change en fonction des trajets." Il faut remarquer que ces déplacements concernent un aliment qui possède un statut particulier: la viande.

Selon le type de commerces, les moyens de locomotion varient: on peut cependant dire que les informateurs privilégient la possibilité d'aller à pied, ce

\footnotetext{
${ }^{11}$ Là encore, les données sur les pratiques des Français rejoignent nos observations: 30\% des ménages continuent de fréquenter les marchés (Régnier; Lhuissier; Gojard, 2006, p. 29).
}

Horizontes Antropológicos, Porto Alegre, ano 13, n. 28, p. 65-86, jul./dez. 2007 
qui n'est pas sans influence sur le rythme d'approvisionnement et son organisation; avec des différences importantes entre habitants des centres villes et banlieusards.

En effet, l'absence de commerces de proximité oblige les ménages de banlieue à recourir à la voiture pour aller s'approvisionner et ils doivent aussi s’organiser plus strictement que les ménages urbains, en particulier par rapport au parcours domicile/lieu de travail, alors que ces derniers n’hésitent pas à ressortir de chez eux après leur retour dans leur domicile. Toutes les familles de banlieue interrogées s’approvisionnent principalement dans les hypermarchés une à deux fois par semaine, en complétant ces courses par des achats faits plus près du domicile quotidiennement (le pain par exemple), sur l'itinéraire lieu de travail/résidence. Ou encore, ils fréquentent parfois un marché le samedi ou dimanche matin, en famille, pour acheter quelques légumes et fruits frais. L'approvisionnement des habitants de banlieue est subordonné plus fortement aux lieux et horaires de travail que les résidents des centres villes. En revanche, les habitants des centres villes peuvent se permettre d'improviser et d'y aller aussi souvent qu'il le faut, puisqu'ils ont des commerces à côté de chez eux. La fréquentation de ces commerces est souvent plutôt pluri-hebdomadaire, ou même quotidienne. Mais le rythme de fréquentation d'un commerce peut être lié à des critères personnels de sociabilité, ainsi les Baudard (Lyon, Croix-Rousse): "Il y marché tous les jours sur le boulevard. On commence à connaître les commerçants. Les producteurs et vendeurs habituels ne sont pas là tous les jours. On choisit donc nos jours. Et le week-end, on évite, c'est envahi et les prix ne sont pas les mêmes”.

Les manières d'organiser les rythmes de fréquentation des divers commerces reposent sur plusieurs critères: proximité; moyens de transports; taille du ménage; horaires de travail; et parfois, liens personnels avec les commerçants. Dans tous les ménages, il y un "rythme de base” qui est donné par la fréquentation soit d'un supermarché de proximité soit d'un hypermarché de banlieue. Ce rythme est au minimum d'une fois par mois et au plus hebdomadaire (le plus souvent par quinzaine). A cette fréquentation se superpose un rythme plus rapide qui est celui des achats de proximité qui peuvent être quotidiens comme le pain ou la viande, mais qui sont dans tous les cas hebdomadaires.

Les femmes ne sont pas, seules, responsables de l'approvisionnement: les hommes y participent, en général munis d'une liste ou alors elles tentent de les “éduquer”, parfois avec succès, comme certaines informatrices nous l'ont 
affirmé... ${ }^{12}$ Néanmoins dans près de la moitié des ménages interrogés, ce sont uniquement les femmes qui prennent en charge l'ensemble de l'approvisionnement. Dans les autres familles, les courses sont réparties dans les couples: les conjoints qui ont des horaires de travail irréguliers ou travaillent moins, s'occupent d'une grande partie des courses alimentaires. Les activités professionnelles jouent ainsi un rôle important dans cette répartition des tâches ainsi que la classe d'âge, mais dans une moindre mesure. Chez les jeunes couples jusqu'à 35 ans, la répartition est indifférenciée et plutôt égalitaire - du moins dans les discours, d'autant plus que l'approvisionnement est quotidien ou presque. Chacun regarde dans le frigidaire ou le placard ce qui manque et va le remplacer. Cependant, le plus souvent, la répartition est organisée et différenciée selon le type de commerces. Si les hommes prennent en charge une partie des courses alimentaires, ils fréquentent surtout les hypermarchés et les marchés. Aux femmes incombent surtout les supermarchés de quartier et les autres commerces plus spécialisés. Il apparaît aussi clairement que les femmes assument le plus souvent la dimension quotidienne de l'approvisionnement et surtout la responsabilité de sa bonne organisation qui leur permet de nourrir leur famille. Leurs conjoints ne vont faire les courses qu'une fois par semaine, ou alors ils ont la charge d'un produit spécifique comme le pain, par exemple, à acheter au retour de leur lieu de travail. Leur statut de "sexe fort" les conduit à acheter le "gros", c'est-à-dire les boissons, les produits de base, nécessairement lourds à transporter. Les hommes qui ont la charge des achats en hypermarché saisissent souvent l'occasion pour les combiner avec ceux de disques ou de livres, parfois en emmenant les enfants pour laisser du temps libre à leur femme; ils organisent ce déplacement d'approvisionnement comme une excursion familiale. Mme Bourdin soupçonne son mari d'aller à l'hypermarché pour contrôler les dépenses du ménage: “C'est mon mari qui fait les courses, il va direct au Carrefour de Chalezeule, une fois par semaine, le samedi matin. Il aime beaucoup son porte-

\footnotetext{
${ }^{12}$ Les courses sont parmi les tâches ménagères, celles auxquelles les hommes participent le plus volontiers: le temps consacré aux achats, toute catégorie confondue, est assuré à 43\% par les hommes (Régnier; Lhuissier; Gojard, 2006, p. 32, citant Brousse 1999). Pour l'approvisionnement alimentaire exclusivement, les femmes s'en chargent dans 58\% des cas, Les autres cas sont répartis à parts égales entre celles faites par les hommes seuls, et celles faites par les deux. Le marché et les grandes surfaces sont des lieux où l'on se rend volontiers en famille, le week-end. Les magasins les plus fréquentés par les hommes sont les boulangeries (Régnier; Lhuissier; Gojard, 2006, p. 33, citant Eymard, 1999).
}

Horizontes Antropológicos, Porto Alegre, ano 13, n. 28, p. 65-86, jul./dez. 2007 
monnaie, il sait que sa femme est très dépensière donc je pense que...”. Cependant, certaines femmes s'arrangent pour y aller à plusieurs afin de rendre la tâche plus agréable. Les choix en matière de répartition des tâches, et ceux des commerces ont une dimension pratique (garde des enfants; horaires de travail irréguliers, etc.). Cependant, à travers ces dernières observations, il semble que la dimension sociale des pratiques d'approvisionnement soit importante, et qu'elle permette de dépasser l'aspect corvée inhérente à une activité contraignante. On ne va pas à plusieurs à l'hypermarché seulement pour avoir plus de bras pour porter les achats, mais aussi parce qu'une tâche faite collectivement prend un tout autre caractère.

Dans son ouvrage sur le "shopping”, Daniel Miller (1998) montre bien les sentiments ambiguës développés par rapport aux courses, principalement par les femmes. Nous retiendrons ici l'idée de combiner ce qui est une "corvée" nécessaire et indispensable, par une certaine compensation qui, d'après nous peut prendre diverses formes, pas seulement celle de se "faire un petit cadeau" en s'achetant quelque chose pour soi (friandise, vêtement, livre, etc.).

L'exemple type de la véritable corvée est l'hypermarché qui doit être menée le plus vite et le plus efficacement possible. Mme Karinski, mère de trois petits enfants: "J'évite au maximum les grands supermarchés, ça je ne supporte pas, donc j'y vais environ une fois par mois pour m'acheter vingt cartons d'eau, dix cartons de lait, c'est tout quoi. Sauf le poisson, point c'est tout”. Mme Karinski y envoie son mari avec les enfants; celui-ci transforme cette corvée en une expérience plaisante, un moment passé avec ses jeunes enfants à qui il offre un repas; il en profite aussi pour "se récompenser" en s'achetant des objets personnels, comme des disques ou des livres. Mme Karinski décrit les achats de son mari:

Lui, les courses, il aime bien; moi, j'aime beaucoup moins parce qu'il dépense des sommes folles! Alors par contre, lui, c’est “Auchan”, “Carrefour”, lui, c’est bing, il a tout sous la main...Il revient avec tout sauf ce qu'on lui a demandé. Il y va le samedi et il prend même les enfants. Ils vont manger dehors, ils font les courses, ils reviennent le soir.

A travers cette description, on perçoit la dimension ludique que prend cette activité dans la mesure où elle ne s’inscrit pas dans ce qui est vécu comme une contrainte pour la plupart des informatrices. L'analyse des entretiens fait apparaître une des stratégies pour rendre les pratiques d'approvisionnement en 
grandes surfaces satisfaisantes: leur donner une dimension sociale en y allant à plusieurs à défaut de pouvoir engager des rapports personnalisés avec les commerçants. Une autre stratégie est de donner à ces achats une signification spécifique, en les inscrivant dans une configuration de magasins. Elle conduit nos informateurs à diversifier les lieux d'acquisition en donnant à chacun d'eux un sens particulier en relation avec une marchandise précise. Cette diversification des espaces marchands peut être plus ou moins sophistiquée: nos interlocuteurs sont précis dans leurs descriptions (tel ou tel produit acquis dans tel ou tel magasin pour des raisons précises) et ils en retirent un certain plaisir, lié à une compétence et une habilité à maîtriser cette "course aux produits”.

Cependant, seuls les petits commerces, surtout ceux de proximité, offrent des relations personnalisées avec les clients. On observe que les consommateurs ont souvent essayé plusieurs commerces avant de fixer leur choix. Les critères de celui-ci mêlent considérations économiques, mais surtout articulent qualité des produits et accueil par le commerçant comme le précise $M$. Chartier: “C’est idiot, mais moi quand j'entre chez un commerçant, j'aime bien qu'il me reconnaisse comme un client du quartier". Cette relation personnelle peut se transmettre d'une génération à l'autre: on va fréquenter le boucher de sa mère, par exemple.

Les conditions que doivent remplir des pratiques d'approvisionnement pour être satisfaisantes, sont les suivantes: l'achat doit avoir un sens par rapport au commerce choisi (prix; qualité; prestige; etc.); la pratique d'achat doit avoir une dimension sociale, voire personnalisée et enfin, elle crée un rapport direct, tangible (y compris la vue, le toucher et l'odorat) avec le produit. Plusieurs conséquences découlent de ces constats: la grande réticence de nos informateurs pour les achats “médiatisés” par différents moyens comme la vente par correspondance ou par internet. Une grande importance est accordée aux commerces de proximité comme permettant de remplir potentiellement toutes les conditions de satisfaction, contrairement, a priori, aux hypermarchés. Cellesci se reflètent également dans les pratiques d'approvisionnement privilégiées, quand le choix existe, mais aussi à travers les discours qui construisent une représentation des commerces et de la sociabilité qui leur est associée. Leur dimension sociale reste un aspect valorisé et valorisateur. Cela nous renvoie, bien sûr, à notre réflexion sur l'insertion des ménages dans leur environnement: "être un client reconnu comme habitant d'un quartier" participe de la construction de l'identité sociale de l'individu. 


\section{Commerces de quartier et sociabilité}

Au-delà d'une sociabilité formalisée dans des associations ou événements particuliers, ces relations de voisinage s'organisent autour des enfants à l'intérieur de l'immeuble - gardes réciproques de jeunes enfants - ainsi qu’à l'extérieur autour de l'école, mais également dans la rue et les commerces. Les pratiques d'approvisionnement participent ainsi de la création du lien social. Les voisins échangent des adresses de "bons” commerçants, ainsi Mme Brustin: “On a un boucher à côté du marché, je ne vais que chez lui. C’est mon boucher exclusif. Je l'ai découvert par mes voisins; ils en parlaient, j’y suis allée et c'est vrai qu'il est très bien”. Certains voisins s'organisent pour aller ensemble dans les hypermarchés; ils peuvent aussi faire des courses les uns pour les autres, ou pour une voisine âgée, devenue impotente. Les relations sociales entre voisins se construisent ainsi souvent autour de l'alimentaire: repas commun; "emprunt" d'un oeuf ou de sel lors d'un oubli; et enfin, achats faits ensemble ou pour quelqu'un du voisinage.

Si les enfants participent grandement de la sociabilité de quartier, leur rôle de lien social se fait aussi en relation avec les pratiques d'approvisionnement. Dès la pré-adolescence, les parents encouragent leurs enfants à aller faire de petits achats alimentaires chez les commerçants de proximité. Cela fait partir d'une socialisation non seulement aux pratiques de consommation, mais aussi à l'espace du quartier: les enfants sont donc incités à fréquenter ces magasins comme partie prenante d'un processus d'apprentissage de l'espace public. ${ }^{13}$ Mme Moullin encourage ses cinq enfants à prendre des initiatives (le plus jeune a 11 ans):

\footnotetext{
${ }^{13}$ V.A. Zelizer (1994) analyse la façon dont, aux Etats-Unis, les enfants ont été évincés de l'espace public. Le résultat est qu'aujourd'hui, aux USA, une enquête montre que les enfants entre 4 ans et 8 ans, issus des classes moyennes, ne sont pas autorisés à sortir seuls du bâtiment, même si celui-ci a une cour. Dans notre cas, il est évident que ce ne sont pas les petits enfants de 4 ans qui sont envoyés faire les courses dans le quartier... Et 7/8 ans semble être l'âge minimum pour sortir seul dans la rue. Néanmoins, les parents français laissent plus facilement sortir leurs enfants, préadolescents et adolescents dans leur quartier, que leurs homologues américains, issus des classes moyennes. L'espace public est encore considéré en France comme un lieu d'apprentissage social.
}

Horizontes Antropológicos, Porto Alegre, ano 13, n. 28, p. 65-86, jul./dez. 2007 
Ils font aussi des achats alimentaires parce qu'il y a des choses qu'ils aiment et surtout ils prennent des goûters énormes qu'ils préfèrent choisir. De temps en temps, ils font la cuisine. Quand ils font ça (acheter de la nourriture) c'est surtout dans les petits magasins du quartier.

Mme Bourdin: "Mon fils a 15 ans cet année; il va faire les courses, il va à la boulangerie, la boucherie.” Le fait que tel ou tel commerçant reconnaisse un enfant comme le fils/fille d'un client et habitant du quartier, est un critère important d'évaluation de celui-ci. Bruno Magnanat décrit combien sa famille se sent bien dans la rue qu'ils habitent à Besançon: "Le marchand de légumes connaît notre gamin...”. Peu à peu, en grandissant, les adolescents gagnent en autonomie en ce qui concerne le choix de leurs achats personnels - vêtements - et les lieux d'achats. Autant que les adultes, voire plus, les adolescents ont leurs quartiers bien à eux, où ils savent qu'ils trouveront des magasins qui correspondront à leur choix stylistique. Un type de configuration commerciale rencontre l'approbation de toutes les générations: les galeries marchandes.

Non seulement, nos informateurs fréquentent et même privilégient les commerces de proximité, ils tiennent aussi des discours sur le rôle de ceux-ci dans la sociabilité de quartier, qui ne correspondent pas toujours à la réalité de leurs pratiques. Ainsi M. Toupard qui ne fréquente pas les commerces de quartier: "Il y a une vie de quartier à Montrapon, là où il y a un peu de commerces. C'est les commerces qui amènent la vie de quartier”. Ou M.Baudry qui vit dans un quartier où les commerces de proximité ont presque disparu:

Les petits magasins de quartier, c'est notre tissu social, c'est la base du tissu. Quand vous allez à Monoprix, vous pouvez toujours discuter avec la caissière, mais elle change tous les jours; là, ils connaissent vos enfants, on les connaît, il y a quand même un autre rapport, ça n’a rien à voir.

Dans son étude d'un quartier londonien, Daniel Miller (2001, 2005) montre la construction faite par la classe moyenne autour des commerces de proximité et de la sociabilité, même si ceux-ci ne sont guère fréquentés car ce voisinage ne correspond pas à leurs aspirations sociales. Dans notre propre recherche, il semble que si les représentations du quartier correspondent aux aspirations sociales et aux modes de vie désirés par les individus, alors ils vont effectivement fréquenter les magasins de proximité, qui pourront jouer un rôle dans une sociabilité de voisinage. D’une certaine manière, ces pratiques 
d'approvisionnement pourront participer à la construction de l'identité des acteurs, comme habitants de tel ou tel quartier ou rue. Deux extraits d'entretiens sont très éclairants:

Moi je suis un peu connu rue Bersot et pas rue des Granges...On connaît le marchand de légumes, on connaît le buraliste, on connaît le marchand de pain, on connaît le marchand de vin...c'est une reconnaissance quelque part. il nous fait 'salut' quand on passe[...]. Moi, j'ai besoin d'une reconnaissance dans mon travail, et pis d'une reconnaissance à l'extérieur...comme habitant du quartier (Bruno Magnanat, Besançon).

M. Chartier de Lyon nous parle de son boucher: “j'aime bien qu'il me reconnaisse comme un client du quartier. Quand un commerçant ne vous reconnaît pas...”[...] Même si c'est plus cher, c'est bien d'être reconnu dans le quartier".

Si l'adéquation entre les commerces de voisinage et les aspirations en termes de mode de vie correspondent moins, les individus ont tendance à aller s'approvisionner ailleurs. ${ }^{14}$ Ainsi les Baudard (Lyon) sont fiers de voir que leurs amis se déplacent pour venir faire leur marché dans leur quartier. En revanche, malgré la présence de certains commerces qui subsistent dans son quartier, Mme Pons (Lyon, Saint-Georges) le décrit comme étant dépourvu de magasins “[...] toutes les épiceries, elles ont été reprises par des petits Tunisiens, c’est ouvert jusqu'à 22h., jusqu'à minuit. Mais il n'y a plus de vie commerçante dans ce quartier, tous les commerces ont disparu”. Ce commentaire permet de comprendre que les commerces "arabes” sont considérés comme des magasins de "dépannage”, ouverts souvent tard le soir et le week-end, ou même comme des "non-commerces". Il serait facile de considérer ces commentaires simplement comme xénophobes, il nous semble plutôt qu'ils sont à mettre en lien avec le statut particulier de ces magasins: ils ne font pas partie des étapes régulières d'une planification des courses; au contraire, ils s'inscrivent dans l'exceptionnel, mais sans proposer de produits spécifiques (ce sont en général des épiceries généralistes). Ils n’entrent donc pas dans le parcours habituel des courses.

\footnotetext{
${ }^{14}$ Il faut remarquer qu'une identité n'est pas monolithique, et que les individus peuvent vouloir exprimer ses diverses facettes à travers différentes pratiques d'approvisionnement, donc de consommation, et à travers des lieux variés.
}

Horizontes Antropológicos, Porto Alegre, ano 13, n. 28, p. 65-86, jul./dez. 2007 
On observe ici combien l'aspiration à un certain mode de vie qui ne correspond pas à la représentation sociale de son propre quartier, conduit un informateur à fréquenter des commerces éloignés de son lieu de résidence (et pas par goût du changement ou de l'exotique...). Miller (2005) constate le même phénomène: les classes moyennes font leurs courses dans des galeries marchandes dans lesquelles on se retrouve "entre soi”. Dans notre étude, ces lieux semblent surtout être privilégiés par les adolescents qui les fréquentent même si ils sont souvent localisées en banlieue ou aux portes de la ville (sauf les Halles à Paris ou la Part-Dieu à Lyon). Cependant, de nombreux informateurs adultes, souvent habitants des banlieues, y vont aussi faire leurs achats vestimentaires. Ces espaces - qui s'apparentent à des non-lieux pour certains chercheurs (Augé, 1992, p. 69-71) - regroupent de nombreuses boutiques qui sont aussi présentes dans les centres villes. Cette présence au centre donne à leurs produits une valeur symbolique ajoutée par l'identité forte de cet espace, qui permet aux magasins de marques similaires de la périphérie de bénéficier d'une sorte de "rappel” (Boukaïa, 1999). D'une certaine manière, ces centres sont des "doubles" des centres villes, mais dont l'esthétique est celui de la modernité et de l'efficacité. Dans une petite ville, comme Besançon, les centres commerciaux comme celui de Chalezeule souvent cité par nos informateurs offrent une alternative commerciale et déambulatoire à la "boucle”. Ils possèdent une dimension familiale à cause de la diversité des choix qu'ils offrent, dans un univers clos, à la température stable et protégé des intempéries (et parfois avec des ouvertures nocturnes prolongées). Ainsi contrairement à sa définition comme non-lieu, le centre commercial et sa galerie marchande procurent du plaisir à ceux qui les fréquentent, même si son affirmation sociale, surtout dans la classe moyenne supérieure, est socialement et culturellement peu admise. Certaines galeries marchandes proposent un "entre-soi" social, ${ }^{15}$ du moins à certains moments de la semaine. Non seulement on y trouvera l'ensemble des magasins qui nous intéresse, mais aussi la clientèle qui est attirée par ceux-ci et qui nous

\footnotetext{
${ }^{15}$ On retrouve cette dimension mais sous une forme extrême dans certaines métropoles extraeuropéennes - nous pensons ici à Bogota que nous connaissons - où les centres commerciaux sont de véritables forteresses sociales, fortement hiérarchisées entre elles et qui s’adressent à des clientèles socialement déterminées. Les études des chercheurs français sur les galeries marchandes sont rares. Les recherches existantes concernent des galeries qui se situent à l'étranger (Amérique du Nord et du Sud).
} 
est donc nécessairement proche socialement, et parfois en termes de classe d’âge. L’idée de parcours, de proximité et de mixité relative des commerces est au cœur des galeries marchandes qui permettent, de plus, de rencontrer ses "semblables" soit en terme social, soit en terme de classe d'âge. Ces caractéristiques expliquent sans doute le succès de ces lieux, certes moins pour des achats alimentaires quotidiens.

\section{Conclusion: un "shopping" à la francaise?}

Notre étude montre que nos informateurs, en particulier les citadins des centres villes, continuent à fréquenter les commerces de proximité. L'appropriation de l'espace du quartier par les habitants se fait à la fois physiquement, parcourir les lieux à pied, mais aussi dans les discours et les représentations. Les commerces de proximité, dans la mesure où il présente une diversité, jouent un rôle dans ce processus, facilité dans notre cas, par le fait que les quartiers où vivent nos informateurs bénéficient d'une image plutôt positive dans la collectivité. Il existe à la fois une sociabilité et une socialisation autour de l'activité d'approvisionnement. "Faire ses courses” crée du lien social, processus dans lequel les enfants prennent une place: dans le quartier, car cette pratique leur permet d'explorer l'espace public de proximité. La définition d'un quartier est liée à celle d'un espace d'interconnaissance réel ou potentiel: pouvoir être salué comme un habitant du lieu, être reconnu par les autres résidents et par les commerçants, constituent autant de preuves tangibles de son appartenance à l'espace du quartier qui se compose souvent d'une rue ou de quelques rues qui peuvent être parcourues à pied. Même s'il n'existe pas de “zoning” social à proprement parler dans les villes que nous avons étudiées, à travers leurs pratiques et leurs représentations, les habitants tendent à délimiter leur voisinage sur des critères sociaux. Bien sûr cela ne conduit pas à des "frontières" spatiales strictes, juste une adaptation des pratiques qui ne peut nécessairement pas réduire la coexistence et la mixité sociales.

La confrontation entre discours et représentations, et les pratiques montrent que les commerces de proximité restent fréquentés et sont valorisés si un certain nombre de conditions sont remplies: diversité de l'offre commerciale, inscription de ceux-ci dans un parcours piétonnier de courses, habituel et souvent planifié d'avance. Les pratiques d'approvisionnement des ménages sont diversifiées et requièrent de véritables stratégies en termes de choix des commerces, d'organisation et de fréquence. Au-delà d'une recherche pragmatique de 
l'efficacité dans leurs stratégies d'approvisionnement, les ménages tentent de trouver une certaine satisfaction dans des pratiques fortement contraignantes. Ce plaisir des achats repose sur plusieurs critères: l'acquisition doit avoir un sens par rapport au commerce choisi (prix; qualité; prestige; etc.); elle doit s'inscrire dans une dimension sociale, voire personnalisée; et enfin, elle doit permettre de créer un rapport direct, tangible (y compris la vue, le toucher et l'odorat) avec le produit.

C'est la dimension sociale qui est bien sûr la plus importante à examiner pour comprendre ces pratiques d'approvisionnement, en lien avec la sociabilité de quartier. C’est la possibilité de créer des liens personnalisés entre commerçant et client, et "d'être un client reconnu comme habitant d'un quartier". Les commerces "font" la vie de quartier pour la plupart de nos interlocuteurs des centres villes étudiés (Paris, Lyon et Besançon), mais pas pour les banlieusards qui doivent se déplacer pour faire leurs courses. Miller (2005) a fait un constat contraire avec ses informateurs londoniens: ils lui tenaient des discours élogieux sur les quelques magasins du quartier, dans lesquels ils n’avaient jamais été, mais qui leur semblaient indispensables au “décor” de leur rue.

En revanche, nos informateurs français, et sauf à vouloir en faire des idéaux-types, ils ne sont pas les seuls en Europe, continuent de fréquenter leurs commerces de quartier, qui existent encore grâce à un urbanisme différent et plus protecteur pour ces commerces. "Faire ses courses" dans les centres villes s'apparente à une pratique "d'esthétique urbaine”. Elle articule encore ancrage territorial ${ }^{16}$ et appartenance à un groupe d'habitants d'un lieu, qui donne à ces centres urbains leurs spécificités urbanistiques et sociales.

\section{Réferences}

AUGE, Marc. Non lieux. Paris: Seuil, 1992.

BOUKAIA, Claire-Anne. Le centre commercial de Chateaufarine: lieu de consommation ou lieu de vie?. In: RAULIN, Anne (dir.). Quand Besançon se donne à lire. Paris: L’Harmattan, 1999. p. 175-188.

\footnotetext{
${ }^{16}$ On peut poser l'hypothèse que cet ancrage territorial des pratiques s'approvisionnement trouve son équivalent au niveau des produits et des cuisines, dans l'importance grandissante accordée au “terroir”, origine et appartenance régionale ou locale.
} 
BOULY DE LESDAIN, Sophie. Château-Rouge, une centralité africaine à Paris. Ethnologie Française, v. 1, p. 86-99, 1999.

BRODY, Jeanne. Le quartier de la rue des Rosiers ou l'histoire d'une cheminement. In: GUTWIRTH, Jacques; PETONNET, Colette (dir.). Chemins de la ville. Paris: Editions du CTHS,1987. p. 85-102.

BROUSSE, Cécile. La répartition du travail domestique entre conjoints reste très largement spécialisée et inégale. In: FRANCE, portrait social. Paris: Insee, 1999. p. 135-151.

CHEVALIER, Sophie. The French Two-Home Project. Materialization of family identity. In: CIEERAD, Irene (dir.). At home: an anthropologie of domestique space. New York: Syracuse University Press, 1999. p. 83-94.

CHEVALIER, Sophie. Etude internationale sur les modes d'habitat: le cas de la France. L'espace du quartier: pratiques d'approvisionnement et sociabilité. Rapport de recherche pour le compte du Plan Urbanisme, Construction et Architecture, Ministère de l'Equipement, des transports et du logement, 2000.

DAMATTA, Roberto. O que faz o Brasil, Brasil?. Rio de Janeiro: Rocco, 1984.

DAMATTA, Roberto. A casa e a rua: espaço, cidadania, mulher e morte no Brasil. São Paulo: Brasiliense, 1985.

DESJEUX, Dominique; MONJARET, Anne; TAPONIER, Sophie. Quand les Français déménagent: circulation des objets domestiques et rituels de mobilité dans la vie quotidienne en France. Paris: Presses Universitaires de France, 1998.

EYMARD, Isabelle. De la grande surface au marché: à chacun ses habitudes. INSEE Première, n. 636, 1999.

GOODY, Jack. Cooking, cuisine and class: a study in comparative sociology. Cambridge: Cambridge University Press, 1982.

HANNERZ, Ulf. Soulside: inquires into ghetto culture and community. New York: Columbia University Press, 1969.

KAPLAN, Steven L. The bakers of Paris and the Bread Question 17701775. Durham: Duke University Press, 1996. 
KAPLAN, Steven L. Good bread is back: a contemporary History of French bread, the way it is made, and the people who make it. Durham: Duke University Press, 2006.

LA PRADELLE, Michèle de. Les vendredis de Carpentras: faire son marché en Provence ou ailleurs. Paris: Fayard, 1996.

LA PRADELLE, Michèle de. La fabrication de la ville au quotidien: espaces marchands, espaces festifs et formes de citadinité. Rapport de recherche ACI Ville, Paris, Ministère de la recherche, 2002.

LALLEMENT, Emmanuelle. Tati et Barbès: différence et égalité à tous les étages. Ethnologie Française, v. XXXV, n. 1, p. 37-46, 2005.

MC KENDRICK, Neil; BREWER, John; PLUMB, John, H. The birth of a consumer society: the commercialization of Eighteen Century England. London: Hutchinson, 1982.

MENNELL, Stephen. All manners of food: eating and taste in England and France from the Middle Ages to the present. New York: B. Blackwell, 1986.

MILLER, Daniel. A theory of shopping. Cambridge: Polity Press, 1998.

MILLER, Daniel. Dialectics of shopping. Chicago: University of Chicago Press, 2001.

MILLER, Daniel. Une rue du Nord de Londres et ses magasins: imaginaires et usages. Ethnologie Française, v. XXXV, n. 1, p. 17-25, 2005.

MINTZ, Sydney W. Tasting food, tasting freedom: excursions into eating, culture and the past. Boston: Beacon Press, 1996.

PERON, René. L'urbanisme commercial à la française. Les annales de la recherche urbaine, v. 78, p. 5-11, 1998.

RAULIN, Anne. L'ethnique au quotidien: diasporas, marchés et cultures métropolitaines. Paris: L'Harmattan, 2000.

REGNIER Faustine; LHUISSIER, Anne; GOJARD, Séverine. Sociologie de l'alimentation. Paris: La Découverte, 2006. 
WHYTE William. F. Street corner society: the social structure of an italian slum. Chicago: Chicago University Press, 1934-1993.

YOUNG, Michael; WILLMOTT, Peter. Le village dans la ville. Paris: Centre de Création Industrielle, Centre Georges Pompidou, 1957/1983.

ZELIZER, Viviana. A. Pricing the priceless child: the changing social value of children. Princeton: Princeton University Press, 1994.

Recebido em 12/12/2006

Aprovado em 16/02/2007 